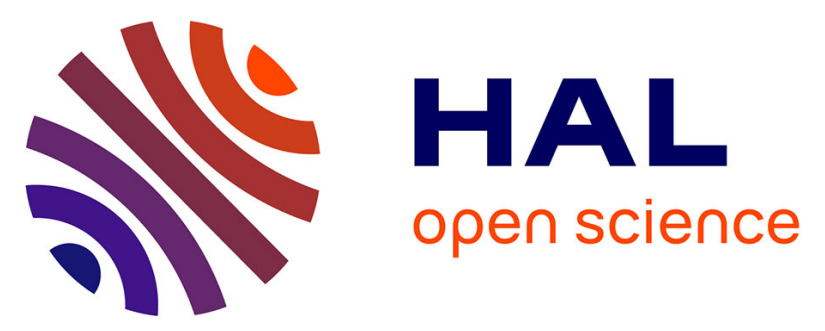

\title{
Liquid-core liquid-cladding optical fibers sustained by light radiation pressure:Electromagnetic model and geometrical analog
}

\author{
Etienne Brasselet, Jean-Pierre Delville
}

\section{- To cite this version:}

Etienne Brasselet, Jean-Pierre Delville. Liquid-core liquid-cladding optical fibers sustained by light radiation pressure:Electromagnetic model and geometrical analog. Physical Review A : Atomic, molecular, and optical physics [1990-2015], 2008, 78, pp.013835/1-7. 10.1103/PhysRevA.78.013835 . hal00384720

\author{
HAL Id: hal-00384720 \\ https://hal.science/hal-00384720
}

Submitted on 15 May 2009

HAL is a multi-disciplinary open access archive for the deposit and dissemination of scientific research documents, whether they are published or not. The documents may come from teaching and research institutions in France or abroad, or from public or private research centers.
L'archive ouverte pluridisciplinaire HAL, est destinée au dépôt et à la diffusion de documents scientifiques de niveau recherche, publiés ou non, émanant des établissements d'enseignement et de recherche français ou étrangers, des laboratoires publics ou privés. 


\title{
Liquid-core liquid-cladding optical fibers sustained by light radiation pressure: electromagnetic model and geometrical analog
}

\author{
Etienne Brasselet and Jean-Pierre Delville \\ Centre de Physique Moléculaire Optique et Hertzienne, Université Bordeaux 1, \\ CNRS, 351 Cours de la Libération, 33405 Talence Cedex, France
}

(Dated: May 18, 2008)

\begin{abstract}
We present an electromagnetic model describing liquid-core liquid-cladding optical fibers sustained by light radiation pressure that encompass the contribution of all the allowed propagating modes. Various sequences of unstable and stable equilibrium liquid column diameters are found above a threshold power. The non trivial relationship between single or multi-valued column diameter with a mono- or multimodal structure of the electromagnetic field is analyzed and the influence of the light-matter interaction geometry is estimated. Moreover, we propose a geometrical interpretation based on a ray optics approach that brings an intuitive understanding of the role played by higher order propagation mode in the occurence of a multistable liquid-core behavior.
\end{abstract}

PACS numbers: 47.10.Ma,42.25.Gy,42.50.Wk,82.70.Kj

\section{INTRODUCTION}

While adaptive optics is now recognized as a well established technique to improve performances of optical systems by checking for distortions and rapidly compensating them using deformable mirrors, lenses or variable index materials [1], it has recently been extended to the conception of new optical systems with variable properties and potential self-adaptation. Using the softness of liquids, advances were first directed toward tunable lensing. On the one hand, externally-driven gradient-index microlenses, such as bulk electro-optical liquid-crystal devices 2] used bulk effects. On the other hand, light focusing has been actuated by liquid interface deformation using electro-wetting 3, 4], radiation pressure (either optical 5] or acoustical 6]) and recently microfluidic devices 7]. Although the actual level of control and reliability of these adaptive liquid lenses found nice applied developments, particularly as active camera lenses for cellular phones, such strategy is almost absent in the case of optical guiding because stabilization of freestanding liquid columns encounters a fundamental limitation associated to the Rayleigh-Plateau instability [8]. A liquid column classically breaks when its length exceeds its circumference due to capillary forces. Strategies based on electric fields [9-11] and acoustic radiation pressure [12] were then prompted to try to stabilize static liquid columns. Unfortunately, the Rayleigh-Plateau onset has been repelled by less than a factor of two, thus preventing any further use in optical systems. A totally different approach, involving flow focusing in microchannels, was implanted recently to bypass the instability and finally build liquid-core/liquid-cladding waveguides [13. This elegant dynamic method offers a good level of stability and control since the refractive indices can be changed with the fluids, and the size and the path of the liquid core can be varied with the fluid flowrates. However, tunability cannot be actuated rapidly, due to the intrinsic inertia of microfluidic systems, and optical guiding is a priori not adapted to the incident light because the size of the liquid core is controlled by liquid flow rates. That is why, up to now, the route followed to build self-adapted optical fibers from the liquid phase has been photopolymerization in order to self-write solid optical waveguides in photosensitive organic materials (see for instance [14]). Although these polymerized fibers are intrinsically selfadapted to the writing beam, the process is not reversible and adaptation is lost as soon as the size and wavelength of the input beam are changed.

A few years ago, an optical technique relying on light radiation pressure [15] has demonstrated that (i) liquid columns with aspect ratio well beyond the RayleighPlateau threshold can be stabilized by a laser wave propagating along the fiber axis, (ii) these columns self-adapt in power and waist of the propagating laser wave and, (iii) self-adaptation continuously adjusts to the beam properties. These tunable optical fibers can furthermore be oriented in any direction by tilting the exciting beam. One can therefore advance the concept of self-adapted liquid step-index optical fiber with total reconfigurability and automatic optimization to the waist and power of the exciting beam. Optically induced liquid columns could thus be particularly efficient to control beam propagation or to optimize light coupling devices because reversibility and self-adaptation considerably reduce the sensitivity to precise mechanical alignments of optical components and to the exact beam properties. Finally, by combining optics and microfluidics, self-adapted liquid waveguides offer a new example of optofluidics system with active optical actuation [16]. However, even if very promising, no theoretical background has been advanced so far to describe the mechanisms at the origin of the optical stabilization and tunability in column diameter and orientation while understanding the involved processes would clearly establish the background necessary for further applications. The purpose of the present paper is to answer these open issues.

In this work, we present an electromagnetic model describing dielectric liquid columns sustained by light radiation pressure that prevents the column to collapse 
through Rayleigh-Plateau instability. Considering the column as a step-index liquid-core/liquid-cladding optical fiber, the light radiation pressure is obtained from the Maxwell stress tensor that encompass the contribution of all the allowed propagating modes. Steady state column diameters depend on light-matter interaction geometry and various sequences of unstable and stable states are found above a threshold power. A single column diameter is predicted when the beam diameter is sufficiently small whereas a multi-valued diameter is expected at larger beam diameter. Although the existence of multistability for the core diameter is unambiguously associated with a multimodal structure of the electromagnetic field, we show that a single valued core diameter can be either obtained when the light field has a mono or multimodal nature. Finally, a geometrical interpretation based on a ray optics approach is proposed and compared to the complete electromagnetic model.

\section{ELECTROMAGNETIC MODEL}

\section{A. General statements}

We consider a dielectric column of liquid 2 in a liquid 1 as a semi-infinite cylinder of radius $R=d / 2$ perfectly centered with an incident linearly polarized [17] (say, along $x$ ) Gaussian beam propagating along $z$, as depicted in Fig. 1(a). The inner and outer refractive indices of liquid optical fiber are $n_{2}$ and $n_{1}$, respectively, with $n_{2}>n_{1}$. This geometry corresponds to the simplest description of the experimental situation explored in 19$]$. In the latter reference, the fluid-fluid interface of a quasicritical phase separated liquid mixture is illuminated by a linearly polarized focused laser beam as sketched in Fig. 1(b). The mixture is enclosed in a $e=1 \mathrm{~mm}$ thick fused quartz cell that is thermally controlled a few Kelvins above the critical temperature, $T_{\mathrm{C}}$, where surface tension vanishes. The phase 2 , of height $0.5 \mathrm{~mm}$, completely wets the cell walls, inducing a wetting layer at the bottom of the cell. At high enough power, typically hundreds of $\mathrm{mW}$, a perfectly beam-centered $0.5 \mathrm{~mm}$-long stable liquid column of phase 2 forms between the interface and the wetting layer [see Fig. 1(b)] following an opto-hydrodynamic instability [20]. A typical image of a light-sustained liquid column is shown in Fig. 1(c). Columns $1 \mathrm{~mm}$-long can as well be stabilized in $2 \mathrm{~mm}-$ thick cells [21].

Without loss of generality, we will further use the parameters that correspond to the experimental study of Ref. [19], which was performed at temperature $T-T_{\mathrm{C}}=$ $2 \mathrm{~K}$. Namely, refractive indices $n_{1}=1.444, n_{2}=1.460$ and surface tension $\sigma=1.75 \times 10^{-7} \mathrm{~N} / \mathrm{m}$ [22]. The intensity profile at $z=0$, i.e. the altitude of the unperturbed interface, is

$$
I(r)=\frac{2 P}{\pi w^{2}} \exp \left(-\frac{2 r^{2}}{w^{2}}\right)
$$

(a)

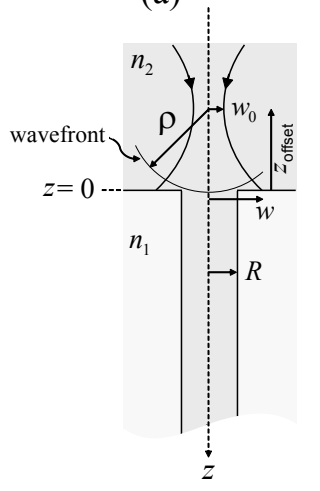

(b)

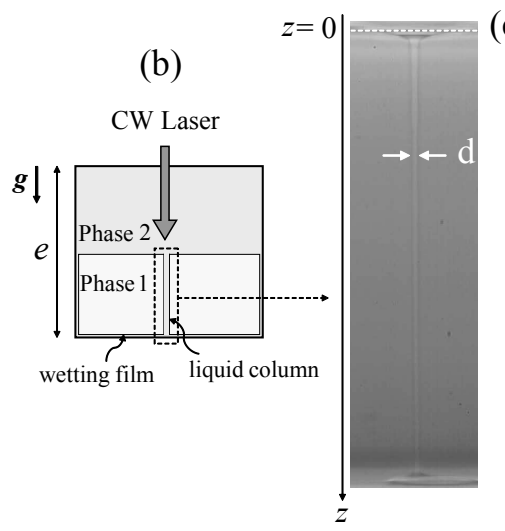

(c)

FIG. 1: (a) Geometry of the problem. (b) Typical experimental situation explored in [19]. The TEM 00 mode of a CW laser is focused at the fluid-fluid interface of the phase separated liquid mixture whose temperature is regulated above the critical temperature $T_{\mathrm{c}}$. The less refractive phase (labeled 1 ) is the denser one and the cell thickness is $e=1 \mathrm{~mm}$. Above a threshold power, a liquid column of phase 2 with diameter $d=2 R$ forms between the interface and the bottom of the cell, as shown in panel (c) where $d \simeq 8 \mu \mathrm{m}$ for $T-T_{\mathrm{c}}=2 \mathrm{~K}$, $w_{0}=1.8 \mu \mathrm{m}$ and $P=600 \mathrm{~mW}$.

where $r=\sqrt{x^{2}+y^{2}}, P$ is the total beam power and

$$
w=w_{0} \sqrt{1+\left(z_{\text {offset }} / z_{0}\right)^{2}}
$$

is the beam radius defined at $e^{-2}$ of maximum intensity. In Eq. $2 w_{0}$ is the beam waist, $z_{0}=\pi w_{0}^{2} / \lambda$ the Rayleigh range and $z_{\text {offset }}$ the offset distance between the plane where is located the beam waist and the fluid interface [see Fig. 1(a)]. The laser wavelength is $\lambda_{0}=514.5 \mathrm{~nm}$ and $\lambda=\lambda_{0} / n_{2}$ is the wavelength in the incident medium (phase 2).

In weightless conditions, the outward optical radiation pressure $\Pi_{\text {radiation, }}$, the inward Laplace pressure $\Pi_{\text {Laplace }}$ arising from surface tension, and the component of the viscous stress normal to the surface must balance on the surface of the column. For a perfectly cylindrical vertical column, the normal component of the viscous stress on the interface vanishes. Thus, the balance of the remaining surface forces is

$$
\Pi_{\text {Laplace }}=\Pi_{\text {radiation }},
$$

which determine the equilibirum solution for a liquid column with radius $R$. On the one hand, the left-hand side of Eq. (3) is merely related to the curvature $1 / R$ of the liquid cylinder as

$$
\Pi_{\text {Laplace }}=\frac{\sigma}{R}
$$

On the other hand the radiation pressure is estimated from the normal discontinuity of the electromagnetic Maxwell stress tensor [23] across the interface. Neglecting the Abraham term oscillating at optical frequency, 
whose time averaging over an optical cycle vanishes, one finds for the time averaged radiation pressure

$$
\begin{array}{r}
\Pi_{\text {radiation }} \underset{r=R}{=}\left\langle\epsilon_{0}\left[\left(n_{1}^{2} \mathrm{E}_{1} \mathrm{E}_{1}^{\top}-n_{2}^{2} \mathrm{E}_{2} \mathrm{E}_{2}^{\top}\right) \mathbf{u}_{r}\right] \cdot \mathbf{u}_{r}\right. \\
\left.-\frac{1}{2} \epsilon_{0}\left(n_{1}^{2} \mathrm{E}_{1} \cdot \mathrm{E}_{1}-n_{2}^{2} \mathrm{E}_{2} \cdot \mathrm{E}_{2}\right)\right\rangle_{t},
\end{array}
$$

where $\mathbf{u}_{r}$ is the unit radial vector, $\epsilon_{0}$ the vacuum permittivity, $\mathrm{E}_{i}$ the real electric field vector in fluid $i,(\ldots)^{\top}$ the transpose and $\langle\ldots\rangle_{t}$ denotes time averaging. Using the boundary conditions for the dielectric fluids 1 and 2 at $r=R, \mathbf{E}_{\mathrm{t}, 1}=\mathbf{E}_{\mathrm{t}, 2}$ and $n_{1}^{2} \mathbf{E}_{\mathrm{n}, 1}=n_{2}^{2} \mathbf{E}_{\mathrm{n}, 2}$, we get

$$
\Pi_{\text {radiation }} \underset{r=R}{=} \epsilon_{0} \frac{n_{2}^{2}-n_{1}^{2}}{4}\left(\left|\mathbf{E}_{\mathrm{t}, 2}\right|^{2}+\frac{n_{2}^{2}}{n_{1}^{2}}\left|\mathbf{E}_{\mathrm{n}, 2}\right|^{2}\right),
$$

where $\mathbf{E}_{\mathrm{t}, i}$ and $\mathbf{E}_{\mathrm{n}, i}$ are the tangential and normal of the complex electric field in the fluid $i$.

Next, the calculation of the field distribution is done by taking into account the waveguiding properties of the liquid column that can be considered as a step-index liquid-core/liquid-cladding optical fiber with radius $R$. Only the propagating modes are further retained and, unless explicitely mentioned, we consider an incident beam whose waist perfectly matches the liquid-liquid interface, i.e. $z_{\text {offset }}=0$.

\section{B. Multimodal structure of the field}

Following $n_{1} \simeq n_{2}$, the propagating modes inside the column are assumed to be the linearly polarized $\mathrm{LP}_{l m}$ modes where integers $\ell \geq 0$ and $m>1$ are respectively related to their polar and radial characteristics 24]. Noting that the incident Gaussian beam is cylindrically symmetric, only the $\mathrm{LP}_{0 m}$ modes are excited. The electric field of the mode $m$ writes (note that the $z$-component can be neglected in the limit $n_{2}-n_{1} \rightarrow 0[24]$ )

$$
\mathbf{E}^{(m)}=E_{0}^{(m)} \mathcal{R}_{m}(r) e^{i \beta_{m} z} \mathbf{u}_{x}
$$

where $E_{0}^{(m)}$ is a constant and

$$
\mathcal{R}_{m}(r)=\left\{\begin{array}{l}
\frac{J_{0}\left(\kappa_{m} r\right)}{J_{0}\left(\kappa_{m} R\right)} \text { if } r \leq R \\
\frac{K_{0}\left(\gamma_{m} r\right)}{K_{0}\left(\gamma_{m} R\right)} \text { if } r \geq R
\end{array}\right.
$$

$J_{n}$ and $K_{n}$ being respectively the Bessel funtion of the first kind and the modified bessel function of the second kind. Moreover $\kappa_{m}$ and $\gamma_{m}$ are the $m$-th roots of the characteristic equation that defines the $\mathrm{LP}_{0 m}$ mode [24],

$$
\kappa_{m} \frac{J_{1}\left(\kappa_{m} R\right)}{J_{0}\left(\kappa_{m} R\right)}=\gamma_{m} \frac{K_{1}\left(\gamma_{m} R\right)}{K_{0}\left(\gamma_{m} R\right)},
$$

with

$$
\left(\kappa_{m} R\right)^{2}+\left(\gamma_{m} R\right)^{2}=V^{2}
$$

where $V=k_{0}\left(n_{2}^{2}-n_{1}^{2}\right)^{1 / 2}$ is often called the normalized frequency and $k_{0}=2 \pi / \lambda_{0}$. Finally, the propagation constant $\beta_{m}$ is defined following

$$
\beta_{m}=\left(n_{2}^{2} k_{0}^{2}-\kappa_{m}^{2}\right)^{1 / 2}=\left(\gamma_{m}^{2}-n_{1}^{2} k_{0}^{2}\right)^{1 / 2} .
$$

The field inside the column is the superpostion of different $\mathrm{LP}_{0 m}$ modes. The weight of the mode $m$ is given by the power transmission of the incident beam into that mode, $T_{m}$, which is expressed as the normalized electric field overlap integral

$$
T_{m}=\frac{\left|\int_{0}^{\infty} \mathbf{E}^{(m)} \cdot \mathbf{E}^{(\mathrm{inc})} r d r\right|^{2}}{\int_{0}^{\infty}\left|\mathbf{E}^{(m)}\right|^{2} r d r \int_{0}^{\infty}\left|\mathbf{E}^{(\mathrm{inc})}\right|^{2} r d r},
$$

where $\mathbf{E}^{(\text {inc })} \propto \exp \left(-r^{2} / w_{0}^{2}\right) \mathbf{u}_{x}$ is the incident Gaussian electric field (recall that we assume $z_{\text {offset }}=0$ ). We obtain

$$
T_{m}=\frac{8 \mathcal{F}_{m}}{w_{0}^{2} R^{2}}\left|\int_{0}^{\infty} \mathcal{R}_{m}(r) e^{-\frac{r^{2}}{w_{0}^{2}}} r d r\right|^{2},
$$

where

$$
\mathcal{F}_{m}=\left[\frac{J_{1}^{2}\left(\kappa_{m} R\right)}{J_{0}^{2}\left(\kappa_{m} R\right)}+\frac{K_{1}^{2}\left(\gamma_{m} R\right)}{K_{0}^{2}\left(\gamma_{m} R\right)}\right]^{-1} .
$$

The transmission coefficient and the total transmission $T=\sum_{m} T_{m}$ strongly depends on the column radius $R$ and beam waist $w_{0}$ as shown in Fig. 2 where $T$ and $T_{m}$ are plotted as a function of $R$ for $w_{0}=1.8$
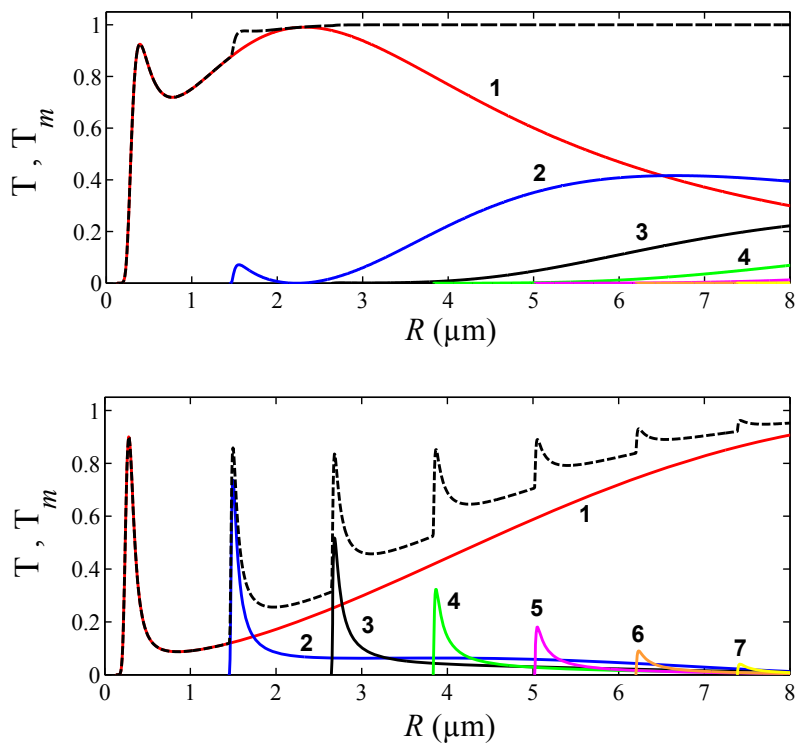

(b)

FIG. 2: (Color online) Total transmission, $T$ (dashed line), and transmission of the mode $m, T_{m}$ (solid lines), vs. column radius for $w_{0}=1.8 \mu \mathrm{m}$ (a) and $7 \mu \mathrm{m}$ (b). The labels (1-7) corresponds to the value of $m$ and the corresponding code color is $m=1$ : red, $m=2$ : blue, $m=3$ : black, $m=4$ : green, $m=5$ : magenta, $m=6$ : orange and $m=7$ : yellow. 


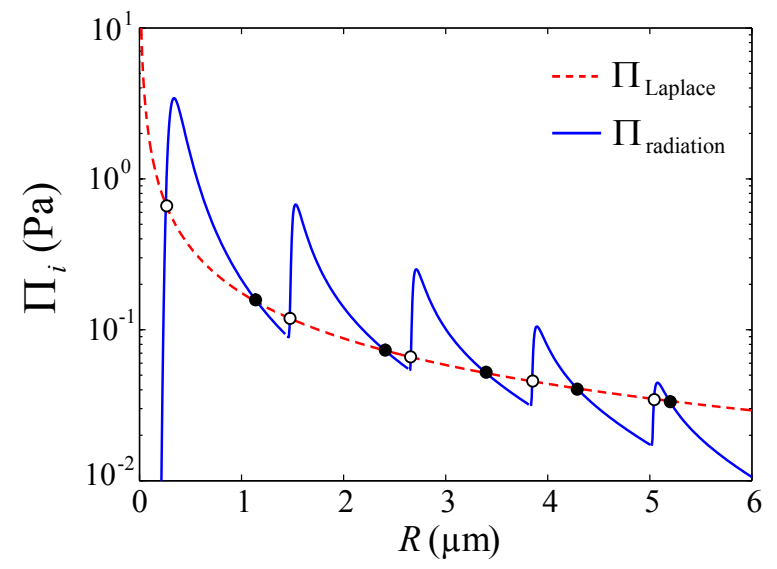

FIG. 3: (Color online) Laplace pressure (dashed line) and radiation pressure (solid line) vs. column radius $R$ for $w_{0}=$ $7 \mu \mathrm{m}$ and $P=500 \mathrm{~mW}$. Filled and open circles correspond respectively to stable and unstable equilibrium radii.

[panels (a)] and $7 \mu \mathrm{m}$ [panels (b)]. Note that only the fundamental mode $\mathrm{LP}_{01}$ always propagates into the liquid fiber, which is a standard result [24]. Higherorder modes $m \geq 2$ appear above cut-off normalized frequencies $V \simeq(3.81,6.97,10.01,13.21,16.33,19.44)$ for $m=(2,3,4,5,6,7)$, which correspond to cut-off radii $R \simeq(1.45,2.65,3.84,5.02,6.20,7.38) \mu \mathrm{m}$ in the present case (Fig. 2). An overall increase of $T$ with $R$ is predicted, however, some peaks can be observed, which correspond to individual modal transmission peaks (Fig. 2). The later peaks are related to mode matching between the incident Gaussian field and the electric field distribution of a mode and are less pronounced for small waists, as observed from the comparison between upper and lower panels of Fig. 2.

From the knowledge of the multimodal structure of the light field propagating into the liquid column, the radiation pressure given by Eq. (6) can now be explicited, which is done in what follows.

\section{Light radiation pressure}

The total radiation pressure exerted onto the column interface results from the combination of all the $\mathrm{LP}_{0 m}$ modes that are allowed to propagate inside the structure. Using the approximated expression for the electric field given by Eqs. (78), Eq. (6) becomes, to the lowest order in $n_{2}-n_{1}$, recalling that $\mathcal{R}_{m}(R)=1$

$$
\Pi_{\text {radiation }}=\frac{1}{2} \epsilon_{0} n\left(n_{2}-n_{1}\right) \sum_{m}\left|E_{0}^{(m)}\right|^{2},
$$

where $n=\left(n_{2}+n_{1}\right) / 2$ is the averaged refractive index. The estimation of the sum in Eq. (15) requires the knowledge of the constants $E_{0}^{(m)}$ of Eq. (7). The later are determined noting that the flux of the $z$-component of the
Poynting vector of the mode $m$ across a plane perpendicular to the $z$-axis equals the power carried by that mode, $P_{m}=\frac{1}{2} \epsilon_{0} n c \iint\left|\mathbf{E}^{(m)}\right|^{2} d x d y$ where $c$ is light speed. Therefore

$$
P_{m}=\frac{\epsilon_{0} n c \pi R^{2}}{2 \mathcal{F}_{m}}\left|E_{0}^{(m)}\right|^{2} .
$$

Finally, combining Eqs. (1516) and using $P_{m}=T_{m} P$, we obtain

$$
\Pi_{\text {radiation }}=P \frac{n_{2}-n_{1}}{\pi R^{2} c} \sum_{m} \mathcal{F}_{m} T_{m} .
$$

\section{Equilibrium states and stability analysis}

By inserting Eqs. (4. 17) in Eq. (3) the equilibirum equation reads

$$
\sigma-P \frac{n_{2}-n_{1}}{\pi c R} \sum_{m} \mathcal{F}_{m} T_{m}=0 .
$$

The column radius $R$ is obtained by numerically solving Eq. (18). No equilibrium radius is found below a critical power $P_{\mathrm{c}}$. Above $P_{\mathrm{c}}$, a discrete set of solutions $\left\{R_{\mathrm{eq}}^{(n)}\right\}$ of radii is predicted. The later situation is illustrated in Fig. 3 for $w_{0}=7 \mu \mathrm{m}$ and $P=500 \mathrm{~mW}$, where the dependence of $\Pi_{\text {radiation }}$ and $\Pi_{\text {Laplace }}$ on $R$ are shown. The stability of steady state solutions is retrieved noting that, in the presence of an infinetisimal displacement $d R \mathbf{u}_{r}$, the total surface force density $\left(\Pi_{\text {radiation }}-\Pi_{\text {Lapace }}\right)_{R=R_{\text {eq }}^{(n)}} \mathbf{u}_{r}$ has a restoring (respectively amplifying) action if $R=R_{\mathrm{eq}}^{(n)}$ is a stable (respectively unstable) solution. The stability criterion thus writes

$$
\left.\frac{\partial \prod_{\text {radiation }}}{\partial R}\right|_{R=R_{\mathrm{eq}}^{(n)}}<\left.\frac{\partial \Pi_{\text {Laplace }}}{\partial R}\right|_{R=R_{\mathrm{eq}}^{(n)}},
$$
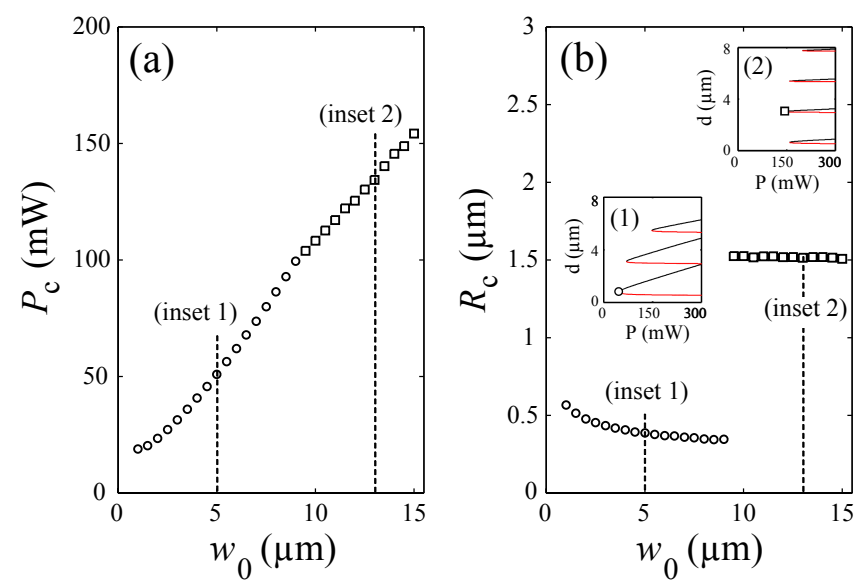

FIG. 4: (Color online) Critical power $P_{\mathrm{c}}(\mathrm{a})$ and radius $R_{\mathrm{c}}(\mathrm{b})$ vs. beam waist. Circles and squares corresponds to the first and the second branch of solutions for the column diameter $d(P)$, as illustrated in insets of panel (b) for $w_{0}=5 \mu \mathrm{m}(1)$ and $w_{0}=13 \mu \mathrm{m}(1)$. 

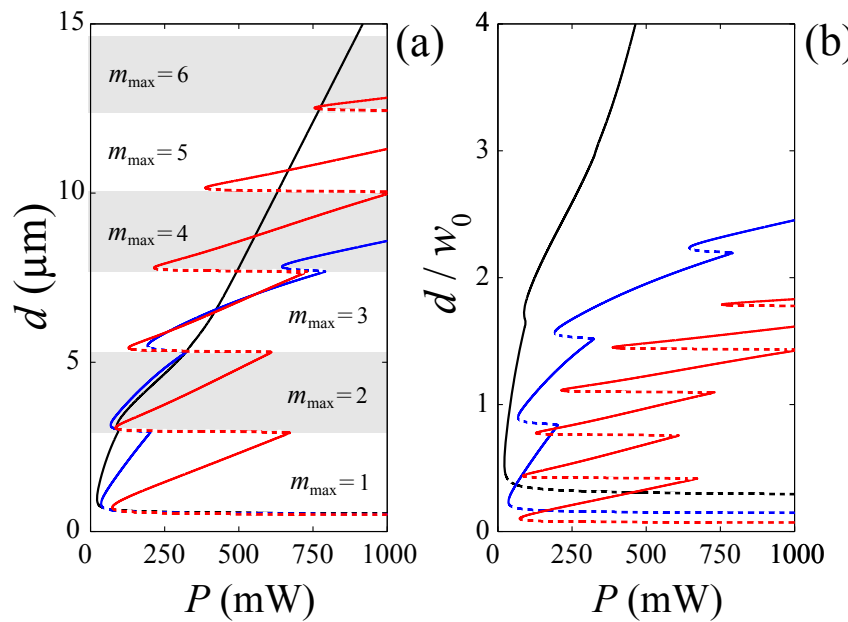

FIG. 5: (Color online) Liquid column diameter $d$ (a) and normalized diameter $d / w_{0}$ (b) vs. power for $w_{0}=1.8$ (black), 3.5 (blue) and $7 \mu \mathrm{m}$ (red). Solid and dashed lines refer to stable and unstable states, respectively. In panel (a) the maximum number of propagating modes, $m_{\max }$, is indicated as alternatively shaded regions.

which is illustrated in Fig. 3, where filled and open circles respectively correspond to stable and unstable equilibrium column radii.

The dependence of the critical power $P_{\mathrm{c}}$ and radius $R_{\mathrm{c}}=R_{\mathrm{eq}}\left(P=P_{\mathrm{c}}\right)$ on the beam waist is shown in Fig. 4. Although $P_{\mathrm{c}}$ increases almost linearly with $w_{0}$ [Fig. 4(a)], $R_{\mathrm{c}}$ exhibits a Plateau-like behavior [Fig. 4(b)] that is related to the branched structure of equilibrium diameters as a function of power. This is shown in the insets $(1,2)$ of Fig. 4(b), which respectively refer to $w_{0}=5$ and $13 \mu \mathrm{m}$.

An overview of equilibrium solutions is displayed in Fig. 5 where the power dependence of the column diameter $d$ and normalized diameter $d / w_{0}$ are respectively presented in Figs. 5(a,b) for $w_{0}=1.8,3.5$ and $7 \mu \mathrm{m}$. Solid (dashed) lines refer to stable (unstable) solutions. For small beam waists (typically for $w_{0}<1.5 \mu \mathrm{m}$ ) a mono-valued diameter is predicted while a more complicated sequence of stable and unstable states is found at larger beam waists. It turns out that each stable branch ending in the plane of parameter $(P, d)$ precisely corresponds to a cut-off radius. In fact, a stable branch is associated to a well-defined number of propagating modes, $m_{\max }$, which is indicated by alternatively shaded regions in Fig. 5(a). Note that a single-valued diameter does not necessarily imply a monomodal structure of the field but merely indicates that higher-order modes contribution to the total light radiation pressure establishes smoothly, as illustrated by Fig. 2(a) where $w_{0}=1.8 \mu \mathrm{m}$.

Next, the influence of an offset between the incident beam waist location and the liquid-liquid interface is explored.
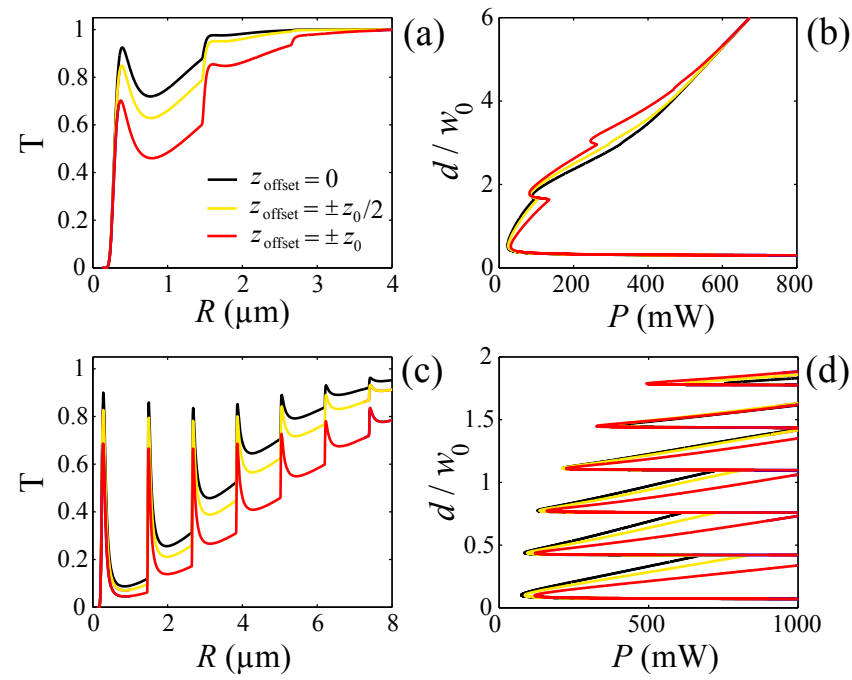

FIG. 6: (Color online) Total transmission vs. column radius and normalized diameter vs. power for $w_{0}=1.8 \mu \mathrm{m}(\mathrm{a}, \mathrm{b})$ and $7 \mu \mathrm{m}(\mathrm{c}, \mathrm{d})$ with different offset values $z_{\text {offset }}$; black: $z_{\text {offset }}=0$; orange: $z_{\text {offset }}= \pm z_{0} / 2$; red: $z_{\text {offset }}= \pm z_{0}$.

\section{E. Role of incident beam offset}

Herafter, we consider $z_{\text {offset }} \neq 0$ [see Fig. 1(a)]. From an experimental point of view, this corresponds to a misalignement of the focal spot of the incident beam with respect to the liquid-liquid interface. Consequently, the modal transmission $T_{m}$ is changed. Indeed, the incident electric field now has an additional dependence in $r$ due to wavefront curvature $\rho$ [see Fig. 1(a)] ,

$$
\mathbf{E}^{\text {(inc) }} \propto \exp \left(-\frac{r^{2}}{w^{2}}+i \frac{n_{2} k_{0} r^{2}}{2 \rho}\right) \mathbf{u}_{x}
$$

where $w$ is given by Eq. (2) and

$$
\rho=-z_{\text {offset }}\left[1+\left(z_{0} / z_{\text {offset }}\right)^{2}\right] .
$$

As expected, Eq. 21 gives a positive curvature when $z_{\text {offset }}<0$, as sketched in Fig. 1(a). The Eq. (12) is thus modified according to the following expression

$$
T_{m}=\frac{8 \mathcal{F}_{m}}{w^{2} R^{2}}\left|\int_{0}^{\infty} \mathcal{R}_{m}(r) e^{-r^{2}\left(\frac{1}{w^{2}}+i \frac{n_{2} k_{0}}{2 \rho}\right)} r d r\right|^{2} .
$$

Then, the equilibrium solutions are obtained by inserting Eq. (22) in Eq. (18). A summary of the results is presented in Fig. 6 that shows the total transmission $T$ and the normalized diameter $d / w_{0}$ for $z_{\text {offset }}= \pm z_{0} / 2$ and $\pm z_{0}$ using $w_{0}=1.8$ [Fig. $\left.6(\mathrm{a}, \mathrm{b})\right]$ and $7 \mu \mathrm{m}$ [Fig. $\left.6(\mathrm{c}, \mathrm{d})\right]$. Note that the result is independent of the sign of $z_{0}$ as expected from the dependence on $\rho$ of Eq. (22).

We conclude that, within an experimentally realistic mismatch $\left|z_{\text {offset }}\right|<z_{0}$, the behavior of the column is not much changed, rendering the experimental validation of the present model [19] almost insensitive to residual misalignment of the incident beam. 

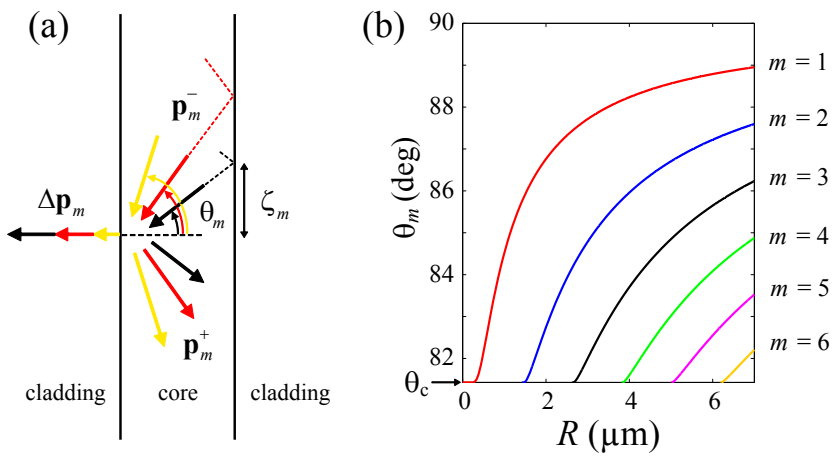

FIG. 7: (Color online) (a) Illustration of the ray optics approach showing the linear momentum transfer $\Delta \mathbf{p}_{m}$ of individual photons of different modes, which are incident at different angles $\theta_{m}$ onto the column interface. (b) Angle of incidence $\theta_{m}$ associated to each mode $m$, the code color being the same as in Fig. 2

\section{GEOMETRICAL INTERPRETATION}

It is known that the light radiation pressure of a plane wave exerted on a flat interface between two dielectrics gives identical results either deriving it from Maxwell stress tensor [22] or ray optics approach [25]. Here, a geometrical interpretation based on the multimodal structure of the light field is particularly desirable in order to bring a more intuitive physical picture than the full electromagnetic approach presented so far, although we are aware that propagating modes into the liquidcore/liquid-cladding optical fiber cannot be considered as plane waves.

For this purpose we consider the ray optics analog of a $\mathrm{LP}_{0 m}$ mode with power $P_{m}$ as a plane wave which is totally reflected at angle of incidence $\theta_{m}$ lying between the critical angle for total internal reflection, $\theta_{\mathrm{c}}=$ $\arcsin \left(n_{1} / n_{2}\right)$, and $\pi / 2$ [see Fig. $7(\mathrm{a})$ ]. By construction we have $\theta_{m}=\pi / 2-\phi_{m}$, where $\phi_{m}$ is defined from the propagation constant given by Eq. (11), namely $\beta_{m}=n_{2} k_{0} \cos \phi_{m}$. The dependence of $\theta_{m}$ as a function of $R$ is shown in Fig. 7(b). The change of linear momentum per photon associated to reflection at the interface is $\Delta \mathbf{p}_{m}=\left|\mathbf{p}_{m}^{+}-\mathbf{p}_{m}^{-}\right|=2 \hbar n_{2} k_{0} \cos \theta_{m} \mathbf{u}_{r}$ [Fig. 7(a)], which can be rewritten as

$$
\Delta \mathbf{p}_{m}=2 \hbar \kappa_{m} \mathbf{u}_{r}
$$

where $\hbar$ is the reduced Planck constant. Within this framework, the radiation pressure for the mode $m$ equals the linear momentum change upon a single reflection per time and area,

$$
\Pi_{\text {radiation }}^{\prime(m)}=2 \hbar \kappa_{m} \Phi_{m}
$$

where $\Phi_{m}$ is the number of photons that reflect on the column wall per unit time and area.

Introducing the number of photons passing through a cross-section $\pi R^{2}$ of the column per unit time, $N_{m}$, and the length between two successive reflections, $\zeta_{m}=$ $2 R \tan \theta_{m}$ [Fig. $\left.7(\mathrm{a})\right]$, we obtain

$$
\Phi_{m}=\frac{N_{m}}{2 \pi R \zeta_{m}}
$$

with

$$
N_{m}=\frac{\alpha_{m} T_{m} P}{\hbar k_{0} c}
$$

where, in the limit $n_{1} \simeq n_{2}, \alpha_{m}=$ $\int_{0}^{R} \mathcal{R}_{m}^{2}(r) r d r / \int_{0}^{\infty} \mathcal{R}_{m}^{2}(r) r d r$ is the fraction of photons inside the core of the liquid optical fiber $\left(0<\alpha_{m}<1\right)$,

$$
\alpha_{m}=\mathcal{F}_{m}\left[1+\frac{J_{1}^{2}\left(\kappa_{m} R\right)}{J_{0}^{2}\left(\kappa_{m} R\right)}\right] .
$$

The total radiation pressure is finally obtained by summing over all the propagating modes,

$$
\Pi_{\text {radiation }}^{\prime}=\frac{P}{2 \pi R^{2} c k_{0}} \sum_{m} \frac{\alpha_{m} \kappa_{m}^{2} T_{m}}{\beta_{m}} .
$$

The radiation pressure expressions obtained within a full electromagnetic approach [Eq. (17)] and its ray optics analog [Eq. (28)] are compared in Fig. 8, where the ratio $\Pi_{\text {radiation }}^{\prime} / \Pi_{\text {radiation }}$ is calculated as a function of $R$ for different values of $w_{0}$. Typically the mismatch is important (as large as 35\%) at small $R$ where diffraction effects becomes significant. Conversely, the relative mismatch is below $10 \%$ above $R \simeq 3 \mu \mathrm{m}$, which corresponds to experimental situations explored in [19] as indicated by the horizontal bars in Fig. 8. In addition, the overall trend at large $R$ shows that the geometrical interpretation is asymptotically valid when the column diameter becomes large compared to the wavelength, as

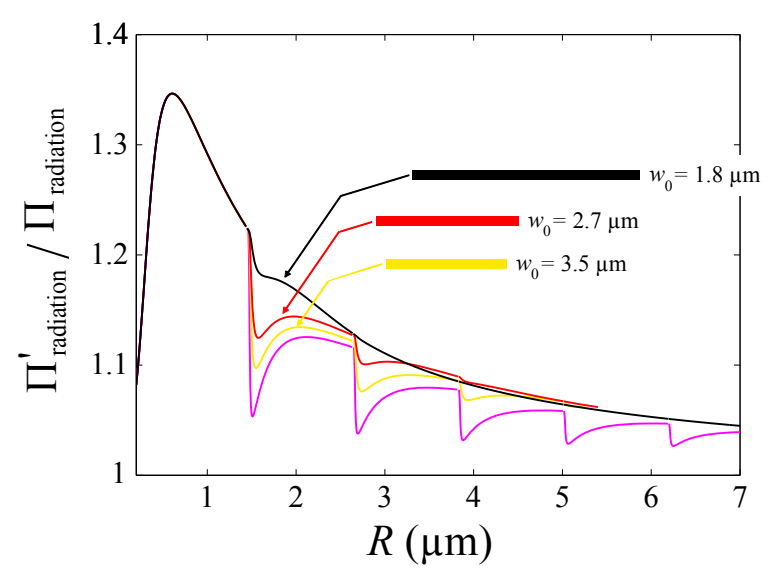

FIG. 8: (Color online) Ratio between the radiation pressure calculated within the geometrical (ray optics) interpretation and the electromagnetic (wave optics) approach vs. column radius for $w_{0}=1.8 \mu \mathrm{m}$ (black), 2.7 (red), 3.5 (orange) and $7 \mu \mathrm{m}$ (magenta). The horizontal bars refers to the experimental range of radii that has been explored in Ref. [19]. 

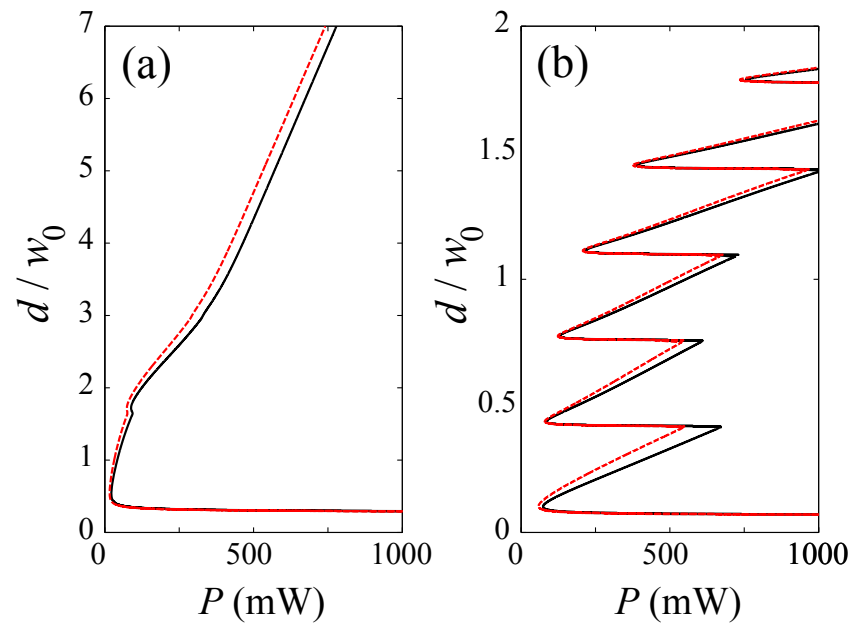

FIG. 9: (Color online) Normalized diameter $d / w_{0}$ vs. power for $w_{0}=1.8 \mu \mathrm{m} \mathrm{(a)} \mathrm{and} 7 \mu \mathrm{m}(\mathrm{b})$ within the electromagnetic approach (solid line) and the ray optics interpretation (dashed line).

expected. As a consequence, only small quantititative changes are found when solving the equilibrium equation using the ray optics approach. This is shown in Fig. 9 where power dependence of the column diameter is calculated for $w_{0}=1.8$ (panel a) and $7 \mu \mathrm{m}$ (panel b) and compared to the previously discussed electromagnetic results.

The geometrical interpretation allows a qualitative understanding of the role played by higher order modes whose corresponding photons ensure a larger amount of linear momentum transfer to the column interface, as illustrated in Fig. 7(a). This can be read from Eq. (23) noting that $\kappa_{m}$ increases with $m$.

\section{CONCLUSION}

An electromagnetic model describing stable stationnary dielectric liquid columns sustained by light radiation pressure is presented. The liquid column is considered as step-index liquid-core/liquid-cladding optical fiber that can either have monomodal or multimodal light propagation behavior. The radiation pressure exerted by light onto the liquid-liquid interface, which defines the liquid fiber core diameter, is calculated using the Maxwell stress tensor and summing the contributions of all the allowed propagating modes. Steady state optical liquid fiber diameters are found when light radiation pressure compensates the Laplace pressure, thus preventing the column to collapse due to surface tension effects. At a given beam waist, equilibrium conditions are fulfilled only above a threshold power that depends on the refractive index contrast and interfacial tension. Above that threshold, a single column diameter is predicted when the beam diameter is sufficiently small whereas a multi-valued diameter is expected otherwise. The stability analysis has evidenced a sequence of unstable and stable equilibrium states whose mapping as a function of the total light power is obtained. Although the existence of a multistable core is unambiguously associated with a multimodal structure of the electromagnetic field, it is shown that a single valued core diameter can be either obtained when the light field has a mono or multimodal nature. Moreover, the role played by the higher order modes of propagation as well as the influence of the coupling geometry were explored. Finally, a geometrical interpretation is proposed. The radiation pressure calculation using a ray optics approach is based on linear momentum transfer of light to the liquid-liquid interface through the total internal reflections of each allowed propagating modes. Comparison with the electromagnetic approach showed its range of applicability and gave a qualitative insight to the predicted multistability.

Considering our theoretical description in close relation to previous experiments (optical stabilisation whatever the aspect ratio and smart tuning in column diameter in the micrometer range), laser-sustained liquid bridges become very promising optofluidic objects to anticipate new optical microsystems based on microfluidics for light guiding and coupling applications or conversely, to build and actuate fluidic micropipes of tunable section (to transfer fluid from one reservoir to another) with optical surface forces.

\section{ACKNOWLEDGMENTS}

We are grateful to Régis Wunenburger for stimulating discussion.

Authors e-mail addresses are: e.brasselet@cpmoh.ubordeaux1.fr and jp.delville@cpmoh.u-bordeaux1.fr
[1] R. K. Tyson, Introduction to adaptive optics, (Bellingham, WA: SPIE, The International Society for Optical Engineering, 2000).

[2] S. Sato, Opt. Rev. 6, 471 (1999).

[3] B. Berge and J. Peseux, Eur. Phys. J. 3, 159 (2000).

[4] S. Kuiper and B. H. W. Hendriks, Appl. Phys. Lett. 85, 1128 (2004).

[5] A. Casner, J. P. Delville, Optics Lett. 26, 1418 (2001).
[6] C. Cinbis, N. N. Mansour, and B. T. Khuri-Yakub, J. Acoust. Soc. Am. 94, 2365 (1993).

[7] S. K. Y. Tang, C. A. Stan and G. M. Whitesides, Lab on a Chip 8, 395 (2008).

[8] J. Eggers, Rev. Mod. Phys. 69, 865 (1997).

[9] H. Gonzalez et al., F. M. J. McCluskey, A. Castellanos and A. Barrero, J. Fluid Mech. 206, 545 (1989).

[10] C. L. Burcham and D. A. Saville, J. Fluid Mech. 40537 
(2000)

[11] M. J. Marr-Lyon et al., D. B. Thiessen, F. J. Blonigen and P. L. Marston, Phys. Fluids 12986 (2000).

[12] M. J. Marr-Lyon, D. B. Thiessen and P. L. Marston, Phys. Rev. Lett. 86, 2293 (2001).

[13] D. B. Wolfe, R. S. Conroy, P.Garstecki, B. T. Mayers, M. A. Fischbach, K. E. Paul,M. Prentiss, and G. M. Whitesides, Proc. Natl Acad. Sci. USA 101, 12434 (2004).

[14] M. Kagami, T. Yamashita, M. Yonemura, and T. Matsui, IEICE Trans. Electron. 90, 1061 (2007).

[15] A. Casner and J. P. Delville, Europhys. Lett. 65, 337 (2004).

[16] D. Psaltis, S. R. Quake and C. Yang, Nature 442, 381 (2006).

[17] Rigorously, a linearly polarized beam leads to liquid column with elliptical cross section. However it is known that the circular symmetry breaking due to non-circularly symmetric polarization state is marginally weak when $n_{1} \simeq n_{2}$ [18], which is the case in the practical situations that have been explored experimentally [19].

[18] A. Casner, J. P. Delville, and I. Brevik, J. Opt. Soc. Am.
B 20, 2355 (2003).

[19] E. Brasselet, R. Wunenburger and J. P. Delville, submitted.

[20] A. Casner and J. P. Delville, Phys. Rev. Lett. 90144503 (2003).

[21] J. P. Delville, A. Casner, R. Wunenburger, and I. Brevik, "Optical Deformability of Fluid Interfaces" in "Trends in Lasers and Electro-Optics Research", Arkin, William T. Ed., Nova Science Publishers (2005); ISBN: 1-59454-4980 .

[22] H. Chrabi, D. Lasseux, E. Arquis, R. Wunenburger and J. P. Delville, European Journal of Mechanics B/Fluids, in press (2008).

[23] L. Landau and E. Lifschitz, Electrodynamics of Continuous Media, Pergamon Press, Oxford, 1984.

[24] K. Okamoto, Fundamentals of Optical Waveguides, 2nd ed. Elsevier, Amsterdam, 2006.

[25] R. Wunenburger, A. Casner, and J. P. Delville, Phys. Rev. E 73, 036314 (2006) 